INPLASY

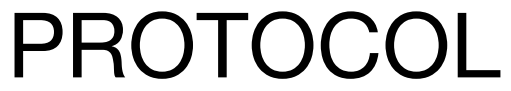

To cite: Huang et al. The Role of Thick acupuncture needles for Soft tissue musculoskeletal injury: A Systematic Review and Meta-Analysis. Inplasy protocol 202220089. doi: 10.37766/inplasy2022.2.0089

Received: 21 February 2022

Published: 21 February 2022

Corresponding author: Huang Jianhao

938396895@qq.com

Author Affiliation:

Shenzhen Hospital of GuangzhouUniversity of Chinese Medicine.

Support: (20181233, 20221328).

Review Stage at time of this submission: The review has not yet started.

Conflicts of interest: Funding comes from Scientific Research Project of Guangdong Provincial Bureau of Traditional Chinese Medicine (20181233, 20221328).

\section{The Role of Thick acupuncture needles for Soft tissue musculoskeletal injury: A Systematic Review and Meta-Analysis}

Huang, J1; Xie, Y2; Feng, J3; Wang, Q4; Liu, Y5; Han, Y6; Hu, C7; Ren, R8; Cui, $X^{9}$.

Review question / Objective: P: Soft Tissue Injuries; I: Thick acupuncture needles; C: Thin acupuncture needles or NSAIDs; O: Efficient or safety; S: randomized controlled trial.

Condition being studied: Common soft tissue injuries among athletes and the general population include rotator cuff tendinopathy, elbow pain,Achilles and patellar tendinopathy, olecranon and pes anserine bursitis, and plantar fasciitis.10 Soft tissue musculoskeletal injury is one of the most common presenting complaints to primary care physicians and is estimated to account for over $50 \%$ of all musculoskeletal injuries reported in the United States annually. The diagnosis and management of such injuries represent a substantial financial burden, estimated at more than USD 15.8 billion annually. Treatments for soft tissue injuries include a wide range of therapeutic modalities, including oral analgesics, injections, physiotherapy, and surgery.Acupuncture is an effective method for the treatment of soft tissue injury, but the influence of the thickness of acupuncture needles on its curative effect is controversial.

INPLASY registration number: This protocol was registered with the International Platform of Registered Systematic Review and Meta-Analysis Protocols (INPLASY) on 21 February 2022 and was last updated on 21 February 2022 (registration number INPLASY202220089).

\section{INTRODUCTION}

Review question / Objective: P: Soft Tissue Injuries; I: Thick acupuncture needles; C:
Thin acupuncture needles or NSAIDs; O: Efficient or safety; S: randomized controlled trial. 
Condition being studied: Common soft tissue injuries among athletes and the general population include rotator cuff tendinopathy, elbow pain,Achilles and patellar tendinopathy, olecranon and pes anserine bursitis, and plantar fasciitis.10 Soft tissue musculoskeletal injury is one of the most common presenting complaints to primary care physicians and is estimated to account for over $50 \%$ of all musculoskeletal injuries reported in the United States annually.The diagnosis and management of such injuries represent a substantial financial burden, estimated at more than USD 15.8 billion annually. Treatments for soft tissue injuries include a wide range of therapeutic modalities, including oral analgesics, injections, physiotherapy, and surgery.Acupuncture is an effective method for the treatment of soft tissue injury, but the influence of the thickness of acupuncture needles on its curative effect is controversial.

\section{METHODS}

Search strategy:

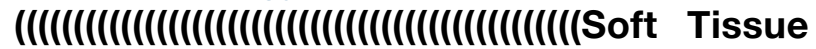
Injuries[Title/Abstract]) OR (Injuries, Soft Tissue[Title/Abstract])) OR (Injury, Soft Tissue[Title/Abstract])) OR (Soft Tissue Injury [Title / Abstract])) OR (Carotodynia[Title/Abstract])) OR (Temporomandibular joint syndrome[Title/ Abstract])) OR (Stylohyoid syndrome[Title/ Abstract])) OR (Omohyoid syndrome[Title/ Abstract])) OR (Muscle contraction headache[Title/Abstract])) OR (Occipital neuralgia [Title/Abstract])) OR (Torticollis[Title/Abstract])) OR (Cervical nerve root impingement[Title/Abstract])) OR (Thoracic outlet syndrome[Title/ Abstract])) OR (Costochondritis[Title/ Abstract])) OR (Xiphodynia[Title/Abstract])) OR (Tietze syndrome[Title/Abstract])) OR (Rotator cuff tendinopathy[Title/Abstract])) OR (Bicipital tendinopathy[Title/Abstract])) OR (Deltoid tendinopathy[Title/Abstract])) OR (Subacromial bursitis[Title/Abstract])) OR (Rotator cuff tear[Title/Abstract])) OR (Adhesive capsulitis[Title/Abstract])) OR (Olecranon bursitis[Title/Abstract])) OR (Lateral[Title/Abstract] AND medial epicondylitis[Title/Abstract])) OR (Cubital tunnel syndrome[Title/Abstract])) OR (De Quervain disease[Title/Abstract])) OR (Intersection syndrome[Title/Abstract])) OR (Carpal tunnel syndrome[Title/Abstract])) OR (Stenosing tenosynovitis[Title) Abstract])) OR (Dupuytren contracture[Title/Abstract])) OR (Trochanteric bursitis[Title/Abstract])) OR (Ischial bursitis[Title/Abstract])) OR (Iliopectineal bursitis[Title/Abstract])) OR (Piriformis syndrome[Title/Abstract])) OR (Meralgia paresthetica[Title/Abstract])) OR (Prepatellar bursitis[Title/Abstract])) OR (Pes anserine bursitis[Title/Abstract])) OR (Patellofemoral syndrome[Title/Abstract])) OR (Iliotibial band syndrome[Title/ Abstract])) OR (Baker cyst[Title/Abstract])) OR (Shin splints[Title/Abstract])) OR (Retrocalcaneal bursitis[Title/Abstract])) OR (Achilles tendinopathy[Title/Abstract])) OR (Plantar fasciitis[Title/Abstract])) OR (Metatarsalgia[Title/Abstract])) OR (Tarsal tunnel syndrome[Title/Abstract])) OR (Morton neuroma[Title/Abstract])) AND ((((acupuncture[Title/Abstract]) OR (electroacupuncture [Title/Abstract])) OR (electroacupuncture[Title/Abstract])) OR (dry-needling[Title/Abstract]))) AND ((( (randomized controlled trial[Title) Abstract]) OR (randomized[Title/Abstract])) OR (placebo[Title/Abstract])) OR (RCT[Title/Abstract])).

Participant or population: Patients with soft tissue injuries Soft Tissue Injuries

Intervention: Thick acupuncture needles.

Comparator: Thin acupuncture needles or NSAIDs.

Study designs to be included: Randomized controlled trial.

Eligibility criteria: Inclusion criteria were (1)Acupuncture needles larger than $0.3 \mathrm{~mm}$ in diameter (2) soft tissue injuries(eg, Achilles/hamstring tendinopathy, and rotator cuff tears), (3)nonsurgical studies, and (4) RCTs.Exclusion criteria: (1) cadaver/nonhuman studies, (2) non-soft tissue indications. 
Information sources: CNKI, SinoMed, WanFang, VIP, pubmed, Embase, Cochrane Library, web of science.

Main outcome(s): Efficient and safety and VAS.

Quality assessment / Risk of bias analysis: Cochrane tool.

Strategy of data synthesis: No heterogeneity selection fixed effects pooled data There is heterogeneity selection, first examine the source of heterogeneity, and then choose a reasonable way to merge, for example, if the source of heterogeneity is statistical heterogeneity, you can choose random effects to merge data.

Subgroup analysis: Divided into subgroups according to the control measures, subgroup 1: fine needles subgroup 2: NSAIDs.

Sensitivity analysis: After deleting the data of any one of the documents, is the result of the merged data of other documents greatly deviated from the original one?

Country(ies) involved: China.

Keywords: Soft Tissue Injuries; Thick acupuncture needles; Efficient; safety.

Contributions of each author:

Author 1 - HUANG JIANHAO.

Author 2 - XIE YUFENG.

Author 3 - FENG JUN.

Author 4 - WANG QIAO.

Author 5 - LIU YI.

Author 6 - HAN YI.

Author 7 - HU CHONGNI.

Author 8 - REN RONG.

Author 9 - CUI XING. 